\title{
OBSERVATIONAL CONCERNS AND TECHNIQUES FOR HIGH-BACKGROUND MID-INFRARED (5 - 20 micron) ARRAY IMAGING
}

\author{
Daniel Y. Gezari \\ NASA/Goddard Space Flight Center \\ Infrared Astrophysics Branch
}

\section{INTRODUCTION}

Broadband observations at 5 - 20 micron with large ground-based telescopes are dominated by thermal background radiation from the telescope optics, instrument and sky. Astronomical sources typically contribute less than one percent of the total detected flux. In most cases the direct, unprocessed image of a bright source (before background subtraction) is indistinguishable from an image of blank sky (Fig. 1). This paper gives an overview of the fundamental concepts, conditions and limitations of high-background mid-infrared imaging. The techniques used to acquire and process the image data are summarized, illustrated with examples from our $58 \times 62$ pixel mid-infrared camera astronomy program.

Bright, mid-infrared astronomical sources are few and far between. If you point a telescope equipped with a near-infrared imaging camera randomly at a thousand places in the sky you are likely to detect some sources (stars) at every position. But if you observe with a mid-infrared camera at a thousand places in the sky you will probably see nothing in a $\sim 1$ arcmin field at every position. There are only a few hundred mid-infrared sources which are bright enough to detect easily, and many of these are well-known standard stars. Altogether in the Catalog of Infrared Observations (Gezari et al. 1993) there are about 2000 mid-infrared sources which are brighter than one Jansky or four mag (our practical detection threshold). But this is a tiny population compared to the enormous number of anonymous and uncataloged near-infrared sources which can easily be imaged from the ground.

The infrared background flux level is determined by the optical throughput (focal ratio of the detector input beam), and by the temperature and emissivity of the telescope optics, the instrument optics, and the sky. Most of the total background comes from the telescope mirrors at a good observatory site. Small changes in the background flux during the observations can contribute to degraded images and dramatically reduced sensitivity. The largest background variations are spatially uniform in the focal plane (due primarily to atmospheric opacity changes) but they can result in second-order spatial defects in combination with subtle linearity and gain effects (primarily in the detector system) or optical efficiency differences (primarily in the telescope) which can become significant after the background is subtracted.

In a typical observatory environment, the thermal background flux in the Cassegrain focal plane of a large ( $\sim$ three-meter) conventional telescope from the $\mathrm{T} \sim 270^{\circ} \mathrm{Kelvin}$ night sky and telescope mirrors is about $10^{9}$ photons $\sec ^{-1} \mathrm{~m}^{-2}$ micron $^{-1} \operatorname{arcsec}^{-2}$ at ten microns. But the detector 
"well" capacity of infrared photoconductor arrays is typically only $10^{5}-10^{6}$ electrons. Thus it would seem that exceptionally short exposures (sampling rates faster than available A/D converters and the time constants array multiplexer circuitry) would be required to avoid saturating the detector. However, the small detector pixels, actual optical efficiency, low photoconductive gain at which our detector can be operated, and reasonably fast sampling rate $(30 \mathrm{~Hz})$ combine to reduce the number of electrons which actually accumulate in each detector well during a $30 \mathrm{msec}$ exposure by about four orders of magnitude, making broadband operation of the large-format array feasible under high-background conditions.

\section{LIMITING NOISE SOURCES}

The sensitivity of broadband mid-infrared observations is limited (ideally) by statistical fluctuations (roughly 1000 electrons per exposure) in the background level (roughly $10^{6}$ electrons per exposure), as compared to detector read noise ( $\sim 100$ electrons per read) and dark current noise ( $\sim$ ten noise electrons per second). Evaluating or specifying signal/noise in an image is little different than doing so for a single detector. But since the individual detector pixels behave very much alike (especially after gain correction of the data), measuring the scatter among adjacent pixels in a featureless area of the image approximates the noise performance of any one of the individual detectors if it were monitored during the total integration interval.

The term "sky noise" is not generally used to describe the background shot noise in the incident photon flux (statistical fluctuations proportional to $\mathrm{n}^{1 / 2}$ ), but rather to describe random variations in the sky emission due to opacity and temperature structure in the atmosphere moving overhead. If the astronomical source of interest and the blank sky could be imaged simultaneously, a nearly perfect sky background subtraction could be made, and effects due to sky opacity structure would be eliminated. Chopping comes close to approximating simultaneous observations, with the chopper frequency determining the degree of temporal coherence.

The frequency spectrum of "sky noise" is another concern. Noise due to opacity differences can have a characteristic $1 / \mathrm{f}$ dependence, the tendency toward long term drifting of the sky emission level, and can be reduced by chopping and sky-subtracting at a sufficiently high frequency (determined by sky conditions). As the chopper beam separation is increased, spatial coherence decreases. But since atmospheric effects occur in the extreme near field, chopper throws would have to become extreme (more than a few arcminutes) for appreciable differences in sky emission between the two chopper positions to become a concern. These effects become aggravated under conditions of poor atmospheric transmission.

Twenty years ago, when single bolometers were commonly used for infrared astronomical observations, fast $(\sim 30 \mathrm{~Hz})$ chopping was standard operating procedure. Changes in the temperature of a bolometer caused by the incident photon flux are measured as changes in bolometer resistance. But bolometers were not easy to use as DC detectors since high resolution $\mathrm{A} / \mathrm{D}$ converters were not then available. Therefore, the difference between two DC voltages (corresponding to the detector temperature at the two chopper beam positions on the sky) was measured using a lock-in amplifier synchronized with the chopper frequency. And since both the bolometers and the sky exhibited noise with $1 / \mathrm{f}$ characteristics, fast chopping was commonly employed with bolometer detector systems. Integrating photoconductor detectors 
and modern A/D converters now make DC measurements with arrays straightforward. The $1 / \mathrm{f}$ current noise of bolometers is not a concern in photoconductor arrays. However, noise is associated with the stability of the electronic system bias and reset levels, and noise performance can be optimized by choice of operating voltages. Appropriate detector operating temperature can minimize dark current noise due to thermal ionization of carriers when the temperature is too high $\left(>15^{\circ} \mathrm{Kelvin}\right.$ ) or loss of responsivity (and consequently degraded signal/noise) due to charge trapping when the temperature is too low $\left(<7^{\circ}\right.$ Kelvin).

\section{BACKGROUND SUBTRACTION AND FLATFIELDING}

A standard set of data reduction procedures is applied to high-background image data. For background subtraction, "reference" images of blank sky (observed simultaneously using the telescope chopping secondary mirror) are subtracted from "source" images. The resulting difference images are flatfielded by dividing each by a normalized blank sky image (a measure of the relative instrumental gain pattern of camera, or the "gain matrix"). Any residual sky signal (background offset level after subtraction) can be removed by subtracting another blank sky image from each difference image (an alternative to nodding the telescope while taking data). After being aligned spatially the flatfielded images are averaged together to improve signal-to-noise and produce a final mosaic.

A single $30 \mathrm{msec}$ camera exposure is referred to as a "frame", and an a co-added series of frames is an "image". A source image $S(x, y, t)$ contains the intensity distribution of the astronomical object $\mathrm{O}(\alpha, \delta)$, an atmospheric background flux component $\mathrm{B}_{\mathrm{a}}(\mathrm{t})$ which is generally featureless but can vary rapidly in intensity, and large background contributions from the telescope $B_{t}(t)$ and instrument $B_{i}(t)$ which change more slowly. A "reference" image $R(x, y, t)$ taken on adjacent blank sky contains everything but the source contribution, although the detected background level could have changed slightly from its value during the "source" observation. The net photon flux is multiplied by the instrument "gain matrix" $g(x, y, t)$, which combines the detector response of each pixel and the optical efficiency of telescope and camera.

$$
\begin{aligned}
& \mathrm{S}(\mathrm{x}, \mathrm{y}, \mathrm{t})=\mathrm{g}(\mathrm{x}, \mathrm{y}, \mathrm{t})\left[\mathrm{B}_{\mathrm{a}}(\mathrm{t})+\mathrm{B}_{\mathrm{t}}(\mathrm{t})+\mathrm{B}_{\mathrm{i}}(\mathrm{t})+\mathrm{O}(\alpha, \delta)\right] \\
& \mathrm{R}(\mathrm{x}, \mathrm{y}, \mathrm{t})=\mathrm{g}(\mathrm{x}, \mathrm{y}, \mathrm{t})\left[\mathrm{B}_{\mathrm{a}}(\mathrm{t})+\mathrm{B}_{\mathrm{t}}(\mathrm{t})+\mathrm{B}_{\mathrm{i}}(\mathrm{t})\right]
\end{aligned}
$$

The basic astronomical image $I(x, y, t)$ is the difference between a source and reference image. If the background contributions are equal in the source and reference images, subtracting them yields the object intensity distribution $\mathrm{O}(\alpha, \delta)$ in the sky, modified by the instrument gain distribution

$$
\mathrm{I}=\mathrm{S}-\mathrm{R}=\mathrm{g}(\mathrm{x}, \mathrm{y}, \mathrm{t}) \mathrm{O}(\alpha, \delta)
$$

The basic flatfielding operation actually occurs in the initial sky subtraction procedure. If any image defects persist after this step, they can be further corrected for by dividing the image by a normalized reference image $R_{o}$ of nearby blank sky, the observationally generated map of net camera response described by $\mathrm{g}(\mathrm{x}, \mathrm{y}, \mathrm{t})$.

$$
\mathrm{O}(\alpha, \delta)=(\mathrm{S}-\mathrm{R}) / \mathrm{R}_{\mathrm{o}}
$$


The reference image used for the division can be the direct sky image obtained simultaneously with the chopper, or a sky frame taken separately under the gain and background conditions as similar as possible. A more rigorous flatfield can sometimes be obtained by dividing by an image composed of the difference between two sky images taken at high and low airmass, which removes the instrument and telescope background contributions from the denominator (in the same way that the corresponding factors in the signal and reference images subtract out in the numerator). But spatial variations in the instrument and telescope backgrounds are usually negligible over time intervals comparable to the integration time, so dividing by a single normalized reference frame usually produces equally good results.

In the raw images shown in Fig. 1, a gradient of about $30 \%$ can be seen across the field due to gain differences among the array pixels. The dark spots are array defects of up to $25 \%$ of the mean resulting from defective bump-bonds between the detector and multiplexer wafers of the hybrid array chip. Despite these gain nonuniformities, the sky-subtracted images can be flatfielded to better than $0.01 \%$ (1s) of the raw image mean. Flatfielding of high-background array images can be the most demanding instrumental calibration requirement. Flatfielding techniques have also been discussed by Hoffmann et al. (1987) and McCaughrean (1987).

Unfortunately, differences in the telescope background, DBt, between the two positions of the secondary mirror can cause a different kind of problem, a spatial offset (or "residual sky") which persists after the sky subtraction and flatfielding process. This offset arises from small differences in the optical path through the telescope optics between the two chopper positions, and is often due to asymmetrical views of the telescope structure, vignetting, etc. This offset can present itself as a constant background level or as an intensity gradient (wedge) across the image. This residual sky background can be removed from an image by nodding the telescope so that the astronomical object appears alternately in the $\mathrm{S}$ and $\mathrm{R}$ images, which are subsequently subtracted from each other in pairs. Or, a single image of nearby blank sky can be subtracted from a number of different object images. The final object image which is co-added from several such corrected images would not limited by the noise level of the single blank sky image, provided that the telescope is repositioned slightly for each integration (to put the object at a slightly different position on the array). When the images are aligned on the common object for averaging, the position of the common sky image becomes shifted in the aligned stack, and the noise is randomized in the average.

Fixed-pattern image defects commonly appear in the image when processing is attempted with a reference image obtained at a different background intensity (Fig. 2). If the source and sky images are not too different, a simple level matching process (adjusting offset and gain factors) can be used to generate a matching reference image. However, large background differences combined with small non-linear detector effects (discussed below) can result in two images which can not be successfully matched. In principle, the same reference image can be used to correct many different data images. However, the background flux and electronic gain of the detector system have to be quite stable on the time scale of the observation.

\section{DETECTOR LINEARITY}

The linearity of the detector response affects more than just the first-order photometric accuracy in imaging array applications. Since most of the charge in the well is generated by background photons, we are always operating at about the same level in the detector well, 


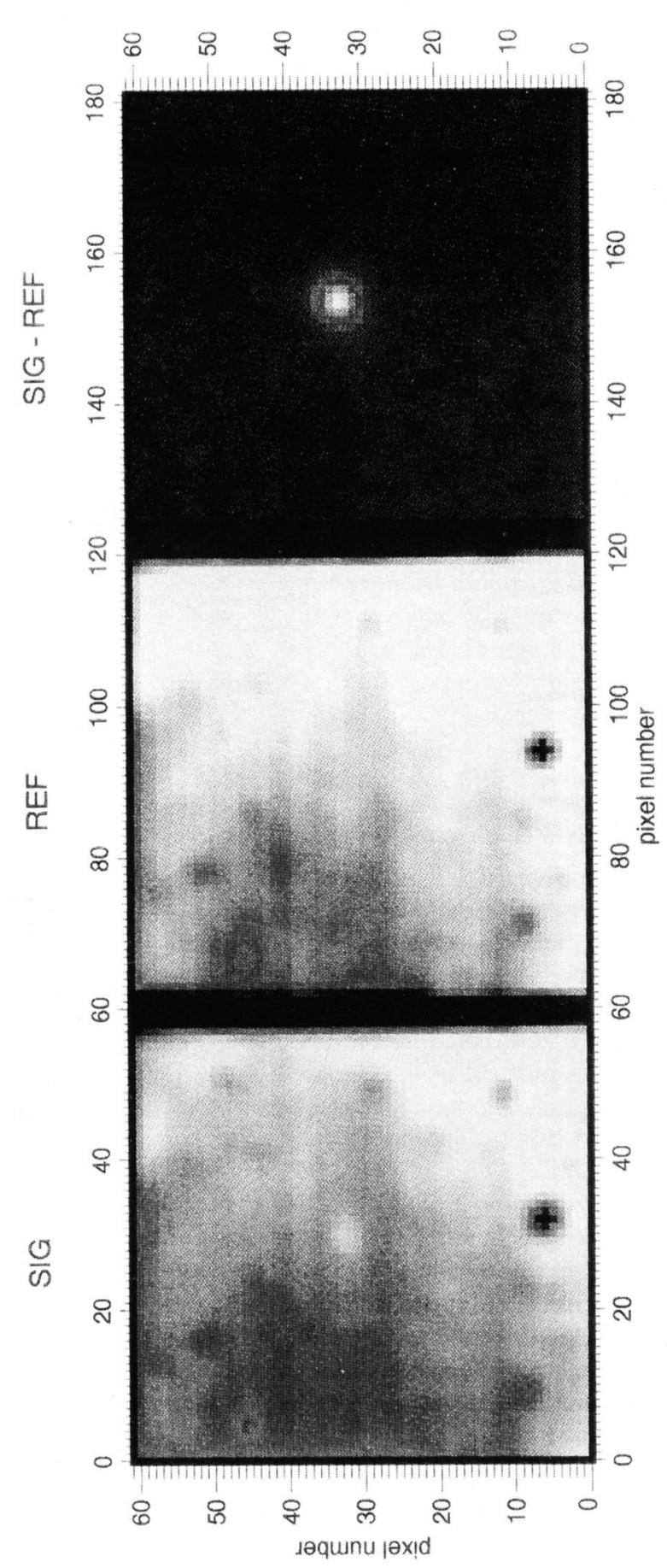

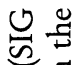

.

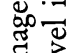

药

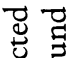

苛

के

के

크

ज

폰

द्न :

0 :

ค

용

क्ष

보ㅇㅛㅛ

옹

을

岁

品

ฮ

घ]

圧要

可

$\checkmark$

잉

눙

过

䆑氙

(1)

幽

.

요

공소

궁

S.

画

- 正.

에에 
22nd REF subtracted trom 30 SIGS - 15 sec increment

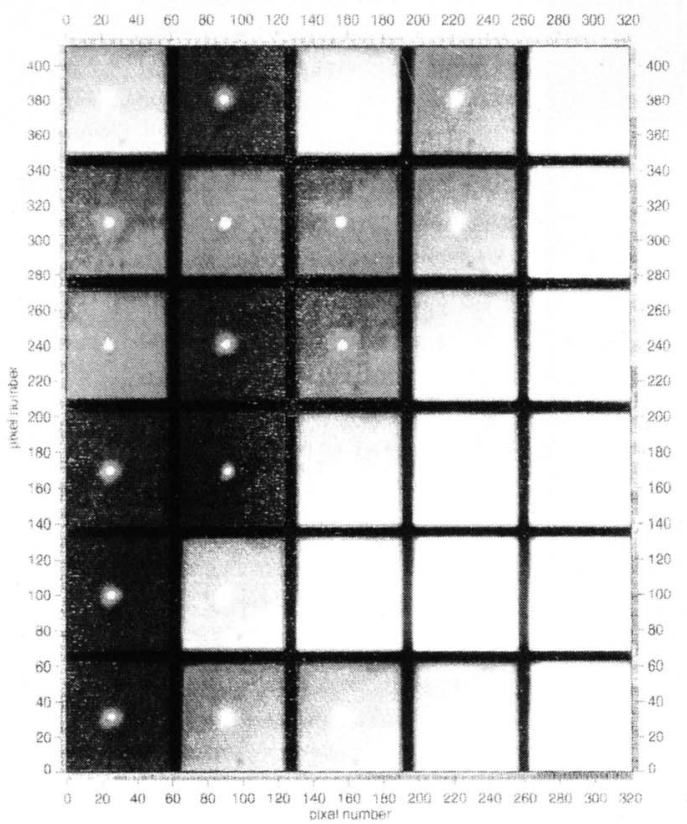

1st fiEF subiracted from 30 SiGs - 15 sec increment

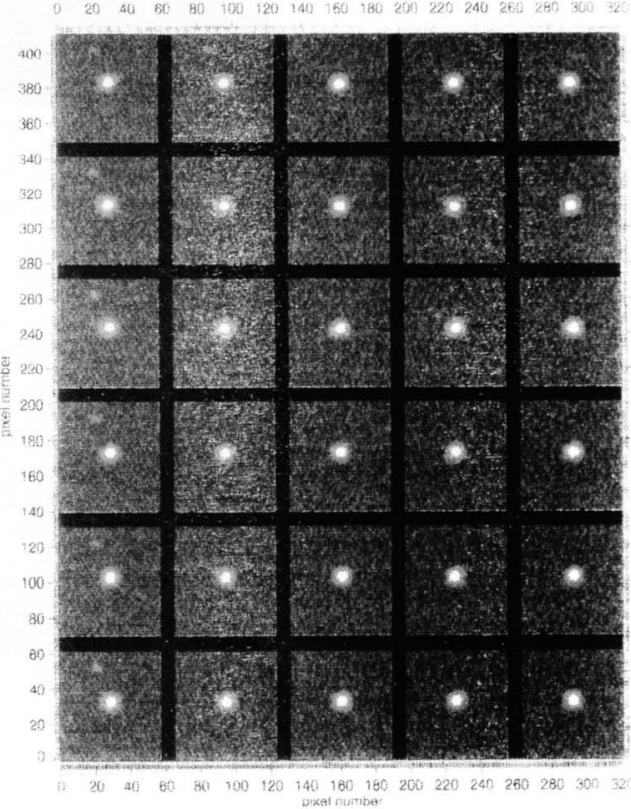

Fig. 2. A comparison of chopped $(3 \mathrm{~Hz})$ and unchopped observations of NGC 1068 on the IRTF with good weather (each with one minute of integration time). The unchopped image shows defects due to the gain matrix response to different background levels, and has about a factor of four worse signal/noise than the chopped image. The unchopped SIG and REF images were taken over an elapsed time of approximately six minutes (a three minutes exposure on the source followed by a three minute exposure on nearby blank sky). The bright point-like nucleus of NGC 1068 has a flux density of about 30 Janskys at 12.4 micron. 
regardless of source strength for all but the brightest sources, and detector linearity would not seem to be a major concern. But very subtle detector problems (latency effects, defective bump-bonds, cross-talk, etc.) become magnified when the background is subtracted and the < $1 \%$ of the well which represents the source flux is expanded to full contrast range in the final image or photometry. In this sense high background imaging is much less forgiving than low-background (where most of the detected signal is due to source photons). Linearity difficulties are aggravated by any changes in system gain or bias levels. Therefore, considerable attention must be paid to the design of a stable detector electronics system.

\section{ARRAY CAMERA SYSTEM}

The infrared array camera system described here was developed for broadband, diffraction-limited 5 - 20 micron astronomical imaging with large observatory telescopes. The camera uses a 58 × 62 pixel gallium doped silicon (Si:Ga) photoconductor array detector manufactured by Hughes/Santa Barbara Research Center (SBRC). The detector array is a hybrid device, assembled from a wafer of Si:Ga detector material (nominally sensitive between 5 - 17 micron), bump-bonded to a Hughes CRC-228 direct readout (DRO) integrated circuit multiplexer chip (Hoffman 1987). The array pixels are read out serially, although the switched FET multiplexer design allows them to be sampled in any order, or polled non-destructively to determine the fullness of the well in low signal/low background applications (with the penalty only of added read noise). A detailed description of the array camera optical and electronic design, detector characteristics, and operating procedures has been presented by Gezari et al. (1992).

One of the very desirable characteristics of this SBRC array is that the photoconductive gain, $G_{p c}$, of the detectors can be adjusted as a function of net detector bias $\left(G_{p c}\right.$ can be reduced to about 0.1 by operating at a net detector bias of four volts). This characteristic of the device permits broad-band operation of the array at higher backgrounds, with no compromise in detected photon noise statistics. Since $G_{p c}$ is a post-detection gain factor, reducing the photoconductive gain reduces the number of electrons generated per incident photon, but does not change the incident photon statistics or signal/noise of the observation.

Under typical high-background conditions the $\mathrm{Si}: \mathrm{Ga}$ array is operated at about $1 / 2$ full-well capacity (full well $\sim 7 \times 10^{5}$ electrons) using a $\sim 30 \mathrm{~Hz}$ frame rate $(30 \mathrm{msec}$ integration time per pixel), and with photoconductive gain set at about 0.1 . This results in noise equivalent flux density NEFD $=0.03 \mathrm{Jy} / \mathrm{min}^{-1 / 2}$ pixel $^{-1}(1 \mathrm{~s})$ with broadband $(\Delta \lambda / \lambda=0.1$, transmission $\sim 80 \%)$ interference filters $\left(1 \mathrm{Jy}=1 \mathrm{Jansky}=10^{-26} \mathrm{Wm}^{-2} \mathrm{~Hz}^{-1}\right)$. The NEFD expressed as noise equivalent brightness is NEB $=0.45 \mathrm{Jy} \mathrm{min}^{-1 / 2} \operatorname{arcsec}^{-2}(1 \sigma)$. The point source NEFD (1s) is about $\sim 0.5$ $\mathrm{Jy} \mathrm{min}^{-1 / 2}$, that is, a $0.5 \mathrm{Jy}$ point source would produce a detector signal equal to the $(1 \mathrm{~s})$ noise in an integration of one minute, since the point source flux is spread over $20-30$ pixels by diffraction and, to a lesser extent, atmospheric seeing. A point source of $\sim 0.1 \mathrm{Jy}$ could be detected in one minute $(1 \sigma)$ if five $\mathrm{x}$ five pixels are binned together in subsequent data analysis. But these performance numbers can be deceiving. Consider an infrared galaxy which has a ten micron flux density of $1 \mathrm{Jy}$ in a point-like (one arcsec) nucleus, and another one Jy distributed in a surrounding uniform disk about ten arcsec in diameter (not an unrealistic model for the brighter infrared galaxies, but a faint source by our standards). The nucleus would be detected with reasonable confidence in about one minute of integration time. But the surface brightness of the disk is 100 times lower, and would require $\sim 10,000$ minutes ( $\sim 150$ hours) of integration 
time to achieve the same signal-to-noise. Binning up five $\mathrm{x}$ five array pixels to an effective 1.3 x 1.3 arcsec synthetic aperture would reduce the integration time by a factor of five to $\sim 30$ hours, a more feasible but still sobering amount of observing time. A low-background, liquid nitrogen-cooled infrared telescope has been proposed for 5 - 20 micron imaging at the South Pole (Gezari 1994), which could greatly improve the efficiency of such observations.

\section{DATA ACQUISITION AND IMAGE ANALYSIS}

The array data frames collected from the source and reference positions of the telescope chopping secondary mirror are sorted and co-added into two algebraic arrays by the data acquisition computer, synchronized with the chopper drive signal. Data are ignored while the chopper mirror is moving between end positions. Two final images with total integration times of typically one minute (1800 $30 \mathrm{msec}$ frames) are down-loaded to the host computer and stored as image pairs.

We have developed an image analysis software package called MOSAIC to process large-format array image data (Varosi and Gezari 1992). The MOSAIC software is now available for use by interested researchers on a limited basis. Fig. 3 shows the Orion BN/KL infrared source complex at 20.0 micron (Gezari and Backman 1995), a 40 x 40 arcsec mosaic image made up of 23 individual overlapping images, to illustrate how large mosaic images of complex fields can be assembled successfully from many individual overlapping array images.

\section{CALIBRATION PROCEDURES}

Array detectors provide intrinsically high relative astrometric accuracy. Astrometric calibration requirements include determination of the array pixel angular size (plate scale) in both dimensions, field rotation and distortion. However, there are several practical factors which complicate astrometric calibration and limit astrometric precision, including telescope tracking, chopper stability, telescope encoder accuracy and mechanical flexure. The telescope magnification also changes slightly with focus setting.

There is a real scarcity of cases where two or more stars fall within a single array field of view (15 arcseconds) and are also easily detected in both the infrared and visible, much less which have relative positions which are measured with high accuracy. Telescope encoder displays are generally not sufficiently precise for sub-arcsecond telescope plate scale calibration measurements. We ultimately determined the array plate scale by programming the telescope to offset reproducibly between the four visible Trapezium stars (which have accurately determined relative positions but which are not bright mid-infrared sources), repeating the programmed motion on a strong, nearby ten micron star, recording multiple exposure infrared images with the array, and measuring positions of the four infrared star images in the infrared data.

For the purposes of absolute flux calibration, images of a standard star must be obtained at least once during the observation of an infrared source, or at least each hour to determine an atmospheric extinction correction at each wavelength. The calibration star images must have signal/noise comparable to the final source images to be calibrated. A dark frame (cold shutter) is useful for instrumental calibration purposes but is not required for reduction of differential (chopped) data if the image contains blank sky. 


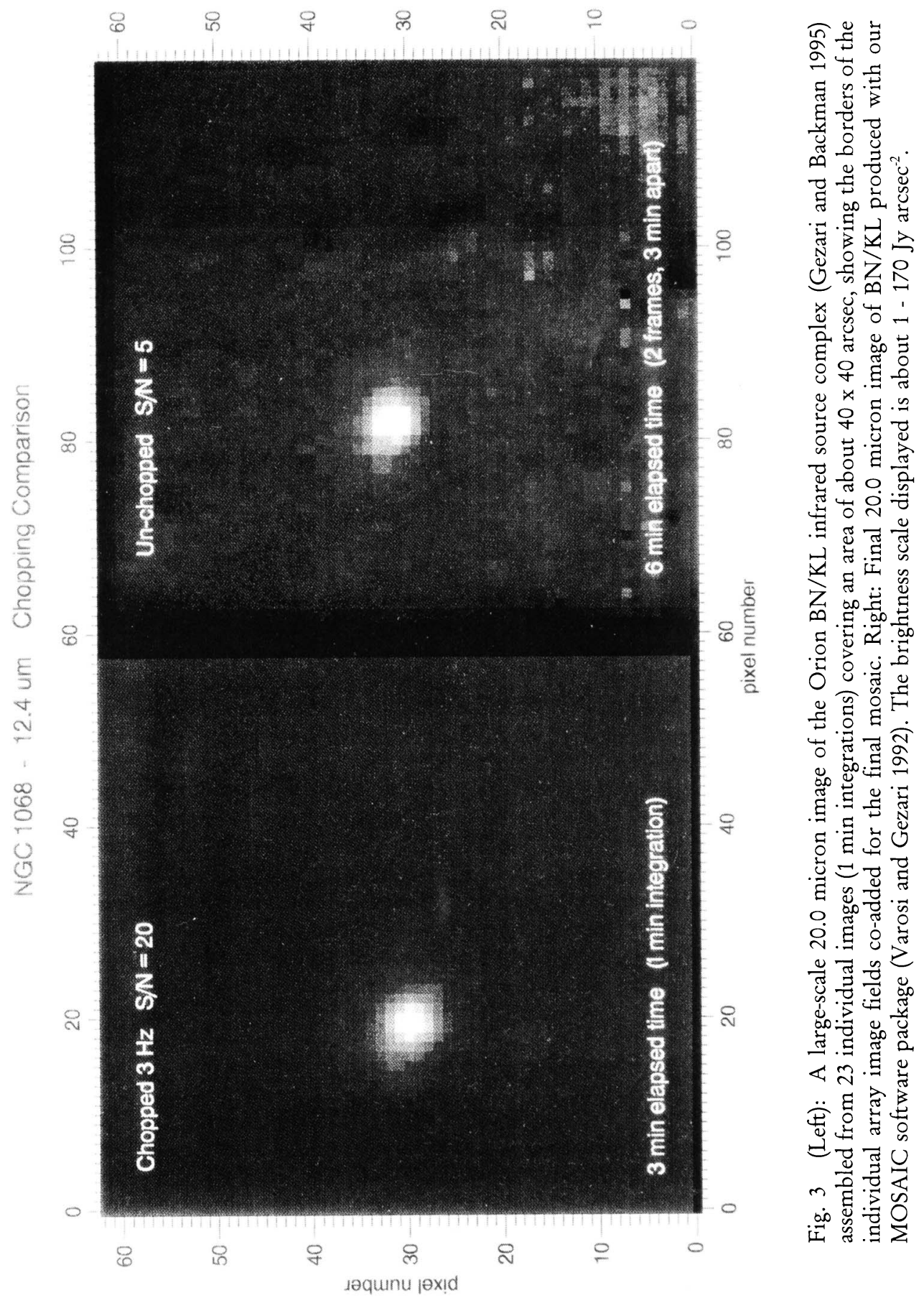


Standard star calibration fluxes have not yet been adopted for all of our mid-infrared (5 - 30 micron) filter wavelengths. The calibration star flux can be extrapolated from observational photometric data for that star found in the list of Bright Infrared Standard Stars compiled at the NASA/Infrared Telescope Facility, or from observations listed in the Catalog of Infrared Observations (Gezari et al. 1993). Since mid-infrared standard star data are generally expressed in relative units (magnitudes), flux densities corresponding to 0.0 mag have to be extrapolated to our filter wavelengths from the mid-infrared absolute flux calibration established by Rieke, Lebofsky and Low (1985), i.e.: at 10.6 micron, $0.0 \mathrm{mag}=36.0+1.2 \mathrm{Janskys}$; at 21.0 micron, $0.0 \mathrm{mag}=9.4+0.5$ Janskys. For additional discussions of mid-infrared calibration standards, see Gillett, Merrill and Stein (1971), Gehrz and Woolf (1971), Tokunaga (1984), and Rieke, Lebofsky and Low (1985).

\section{CONCLUSION}

One of the most frequently asked questions is whether chopping is necessary with mid-infrared array detectors. Sky subtraction and flatfielding operations seem to break down on time scales of roughly one minute when making staring (unchopped) observations under average weather conditions. Of course, the ability to observe without chopping would provide significant practical advantages. High speed secondary mirror chopper mechanisms would be not be needed (a concern in the design of larger telescopes) and image quality and astrometric accuracy would not be limited by chopper positional stability.

If some part of an image is known to contain blank sky, and an accurate gain matrix exists for that image, the two can be used to create a synthetic sky frame for background subtraction without chopping. Sky background subtraction can also done by "dithering" (shifting the position of the source on the array in two images), then subtracting the shifted images, and deconvolving the source structure (a process similar akin to nodding). In practice, sky subtraction using these alternate methods can be rather tedious, and does not treat the high frequency noise components.

Nodding the telescope (moving alternately between two positions in the sky) can be effective for sky subtraction but has some limitations since nodding is slow, and may not allow for sky subtraction to minimize $1 / \mathrm{f}$ sky background variations. Low frequency changes in the sky background are generally largest, and this $1 / \mathrm{f}$ behavior of the detected sky flux level means that sky noise components below the chopping frequency can be significantly reduced. At some point chopping faster, at the high cost of mechanical complexity, stability, heat dissipation, vibration, and image quality, will not result in significant noise reduction.

At very good, dry observatory sites the $1 / \mathrm{f}$ noise component can become negligible at low frequencies, and $1 \mathrm{~Hz}$ chopping seems to be adequate for minimizing noise associated with atmospheric emission variations. However, at sites with poor atmospheric conditions, faster (ten $\mathrm{Hz}$ ) chopping could provide a significant advantage. Chopping against blank sky provides real-time sky background subtraction and data for gain matrix correction at a reasonable cost in observing efficiency. Depending on weather conditions, chopping may not always be required for routine high-background array observations. But chopping can always improve the limiting sensitivity achievable for imaging faint sources.

We are grateful to Mary Hewitt of Hughes/Santa Barbara Research Center (SBRC) for her 
expert guidance during the development of the array camera system. We acknowledge stimulating discussions with Dick Joyce, Harvey Moseley and Frank Varosi. We thank Michael Hauser at NASA/Goddard for his on-going support of this program. This research is funded by NASA/OSSA (RTOP 188-44-23-08).

\section{REFERENCES}

Gehrz, R. D. and Woolf, N. J. 1971 Ap J 165, 185

Gezari, D. Y. 1994 Proposal for Directors Discretionary Fund Support, NASA/Goddard Space Flight Center, (preprint)

Gezari, D. Y. and Backman, D. E. 1995 (submitted to Ap.J)

Gezari, D. Y., Folz, W. C., Woods, L. A., Wooldridge, J. and Varosi, F. 1992 Proc. SPIE, 973, 287

Gezari, D. Y., Schmitz, M., Pitts, P. and Mead, J. L. 1993 Catalog of Infrared Observations, NASA RP-1294

Gillett, F. C., Merrill, K. M. and Stein, W. A. 1971 Ap J 164, 83

Hoffman, A. W. 1987 Proc. Hilo Detector Workshop, Univ. of Hawaii, p. 29

Hoffmann, W., Fazio, G. G., Gezari, D. Y., Lamb, J., Shu, P. and McCreight, C. 1987 Proc. Hilo Detector Workshop, G. Wynn-Williams and E. E. Becklin, eds., Univ. of Hawaii

McCaughrean, M. J. 1988 Ph.D. Thesis, University of Edinburgh

Rieke, G. H., Lebofsky M. J. and Low, F. J. 1985 AJ 90, 900

Tokunaga, A. T. 1984 AJ 89, 172

Varosi, F. and Gezari, D. Y. 1992 Astronomical Data Analysis Software and Systems. II., R. J. Hanisch, R. J. V. Brissenders and J. Barnes, eds., ASP Conf. Series 52, 393

\section{DISCUSSION}

D'ODORICO: Whether chopping is needed for infrared arrays at large telescopes and if so, at which frequency is a relevant question for the engineering of the secondary mirror units of these telescopes. Do you know at which chopping frequency it is foreseen to operate the midinfrared camera at the Keck telescope?

McLEAN: The mid-infrared camera and the long wavelength spectrometer for Keck have not yet been commissioned. Nevertheless, the $\mathrm{f} / 25$ infrared secondary is a chopping secondary and it has been used with the near infrared camera. I do not know what frequency will be used ultimately at $10-\mathrm{m}$, but the secondary is capable of fast chopping.

FINGER: Measurements in the mid-infrared consistently show that noise is decreasing with increasing chopping frequency.

GEZARI: It is not clear that going to chopping frequencies faster than about $1 \mathrm{~Hz}$ results in an improvement in noise associated with the sky emission. Longer term transparency variations seem to dominate, suggesting that fast nodding may be a viable alternative to chopping in large telescopes. But the question is open and more work needs to be done on this.

MOSELEY: What chopping frequency was required to reduce noise to background limit?

GEZARI: In La Silla at the 2.2-m telescope (IRAC1) and the 3.6-m telescope (TIMMI) noise 
measurements have been performed in the $\mathrm{L}$ and $\mathrm{N}$ bands. The noise is decreasing with increasing chopping frequency. Depending on the atmospheric conditions background limited performance is achieved at frequencies between a few $\mathrm{Hz}$ and $10 \mathrm{~Hz}$. 